\title{
Methodology for Determining of Generator Operation Point for Ensuring Voltage Stability Against Generator Faults in Jeju-Haenam HVDC System
}

\author{
Sang-Gyun Kang*, Sangsoo Seo*, Byongjun Lee ${ }^{\dagger}$ and Joon-Young Joo**
}

\begin{abstract}
This paper presents a new algorithm for determining generator operation point for maintaining stability considering generator faults in Jeju-Haenam HVDC system. As the HVDC system consumes reactive power for the transmission of active power substantially, compensation of reactive power is essential. And the HVDC system is operated on frequency control mode. That is to say, the HVDC system almost manages system frequency. Therefore, we recognized that the Jeju system could be unstable if the reactive power consumed by the HVDC is insufficient when out-of-step occurs with large generators. When the solution of power flow analysis does not converge due to the unstable system phenomenon, we have difficulty in establishing countermeasures as the post-fault information is not available. In this paper, for the purpose of overcoming this difficulty in establishing countermeasures, we introduce the $\mathrm{CPF}$ (Continuation Power Flow) algorithm. This paper suggests an algorithm for calculating the output limitation of the generator to maintain the stability in case of generator fault in the Jeju system.
\end{abstract}

Keywords: Voltage stability, Current-based HVDC, The Jeju Power System, Generator Contingency, $\mathrm{CPF}$ (Continuation Power Flow)

\section{Introduction}

The Jeju power system is an isolated island. Although the load demand is increasing more and more, it is difficult to construct additional thermal generators in the Jeju, and the generation cost in the Jeju system is more expensive than the mainland system. To solve abovementioned problem, the 300MW CSC (Current Sourced Converters)-based HVDC system was installed between the Haenam substation on the mainland and the Jeju substation on Jeju Island in 1998 [1]-[3].

Fig. 1 represents the Jeju system. The total peak load of Jeju Island is approximately $500 \mathrm{MW}$, and $150 \mathrm{MW}$ is transmitted over HVDC under normal condition. As of 2006, major generators in the Jeju system are two units (100MW) in the South Jeju bus and one unit $(75 \mathrm{MW})$ in the Jeju bus. These three generators and the transmitted power through HVDC line supply most of the loads in Jeju Island. As HVDC consumes reactive power for the transmission of active power substantially, compensation of reactive power in the system is essential. Actually, reactivepower compensation is carried by filters and synchronous condensers ( 2 x 55MVar) in the Jeju system.

Table 1 shows the HVDC operation criterion, which is recommended by the KPX (Korea Power eXchange; Inde

$\dagger \quad$ Corresponding Author: Dept. of Electrical and Electronic Engineering, Korea Univerity, Korea.(leeb@korea.ac.kr )

* Dept. of Electrical and Electronic Engineering, Korea Univerity, Korea.( riversg78@korea.ac.kr and sance97@korea.ac.kr)

** KPX (Korea Power eXchange), Korea .(zoo@kpx.or.kr )

Received : June 18, 2009; Accepted : December 30, 2009

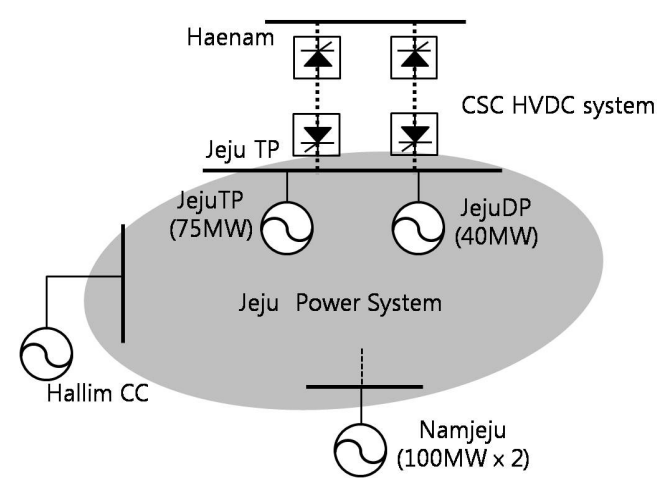

Fig. 1. The caption for a figure must follow the figure.

Table 1. The caption must be followed by the table

\begin{tabular}{|c|c|c|c|}
\hline \multirow{2}{*}{ classification } & \multicolumn{2}{|c|}{ Normal condition } & \multirow{2}{*}{$\begin{array}{c}\text { Abnormal } \\
\text { condition }\end{array}$} \\
\hline & Above $300 \mathrm{MW}$ & Below 300MW & \\
\hline $\begin{array}{c}\text { Power transfer via } \\
\text { HVDC }\end{array}$ & $150 \mathrm{MW}$ & $50 \%$ of loads & $\begin{array}{c}\text { Frequency } \\
\text { control }\end{array}$ \\
\hline
\end{tabular}

pendent System Operator), in detail. According to Table 1, the HVDC system operates in frequency control mode. That is to say, the HVDC system manages system frequency in the Jeju. If generators have an accident in the Jeju AC system, the HVDC system would guarantee active power balance.

The Jeju system is highly dependent on the HVDC system and large generators. Therefore, when the HVDC system or a large generator has unexpected problems, it has a fatal effect on the stability of the Jeju system. On April 1, 2006, the entire Jeju system was blacked out because of the 
failure of the HVDC system and the generator cascadedoutage. As a result of this blackout, many researchers have taken an interest in the Jeju system and have analyzed the Jeju system stability in detail.

Many contingencies such as transmission line, generators, and HVDC system, were analyzed. The system was stable in case of faults in transmission line and HVDC system. Especially, the problem of voltage instability in ACDC systems is generally concerned with strength of the AC system, which is determined by ESCR (Effective Short Circuit Ratio) at the converter bus [4]-[6]. The result of ESCR analysis was that the minimum value is larger than 5 . Therefore, we consider that the Jeju system is stable in respect to ESCR. As the Jeju-Haenam HVDC system is operated on frequency control mode, the HVDC system maintains the system frequency when out-of-step occurs with large generators in the Jeju system. As there is an extra margin of $150 \mathrm{MW}$ up to the maximum rated capacity of the HVDC, the HVDC is capable of supplying 100MW additionally in the event of fault with a 100MW-rated generator. However, it was recognized that the Jeju system could be unstable if the reactive power consumed by the HVDC is insufficient for the Jeju system.

When the solution of load flow analysis does not converge due to the unstable system phenomenon, we have difficulty in establishing countermeasures as the post-fault information is not available. For the purpose of overcoming this difficulty, introduction of the CPF algorithm is intended in this paper. In the past few decades, various methods of analyzing the voltage stability using the CPF algorithm have been presented [7]-[12]. The major feature of the CPF algorithm is that singularity of Jacobian matrix can be avoided by reformulating the power flow equations and applying a locally parameterized continuation technique [7]. In [9], Flueck had presented a tool estimating the severity of contingency by obtaining the solution path approaching outage point with branch-parameterized CPF method. This tool benefits to evaluate, particularly, tough contingencies where traditional power flow solvers cannot converge.

Presentation of an algorithm for calculating the output limitation of the generator to maintain the stability in case of generator fault in the Jeju system, where HVDC is operated in constant-frequency mode, is intended in this paper. For this purpose, the new method that parameterizes the critical generator output is presented.

In Section II, the basic concept on proposed method and system modeling for application of CPF is explained. In Section III, the result of $\mathrm{g}-\mathrm{V}$ analysis on the peak system of Jeju in 2006 is described. In Section IV, the conclusion is given.

\section{The Proposed Method}

\subsection{Basic Concept}

Consider two power systems linked with the HVDC sys- tem that operate in frequency control mode, as shown in Fig. 2. When disturbance, such as generator outage or load increase, occurs, the HVDC system guarantees the frequency of system $B$ by increasing transfer power from system A to system B. Assume that system A is robust enough to ensure against any disturbance in system B. If loss of active power sources is less than the maximum power transfer capability of HVDC in system B, we don't need to consider frequency problem. Unfortunately, the more power is transmitted by HVDC, the more reactive power is consumed from system B through HVDC. Therefore, If reactive power required for HVDC power transmission is not sufficient in system B, this system B will face serious problem even when the maximum capacity of HVDC is not exceeded.

Fig. 3 shows the basic concept of the proposed method. The power that HVDC can additionally transmit is limited not by the capacity of HVDC device, but by the capacity of supplying reactive power in system B. Figure 3 is the result of applying continuation power flow on the locally parameterized output $\mathrm{P}$ of the generator. Y-axis indicates system voltage and $\mathrm{X}$-axis indicates shifted active power from the faulted generator to the HVDC. PG0 means the generator

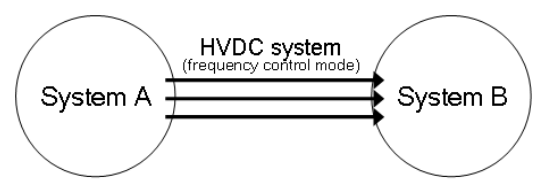

Fig. 2. Two power systems with HVDC that operate in frequency control mode.

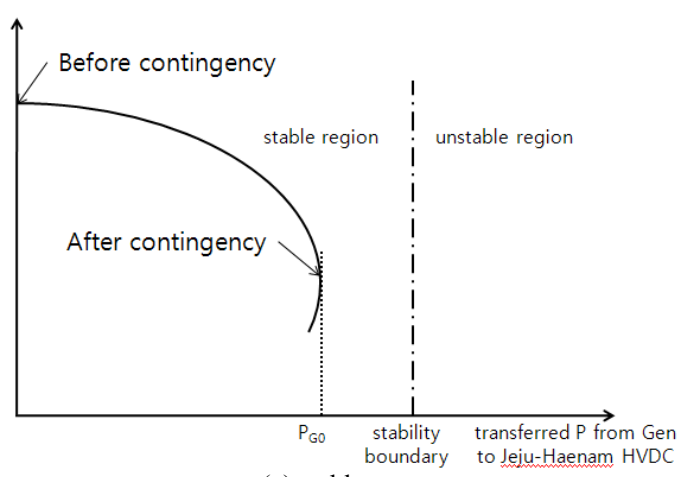

(a) stable case

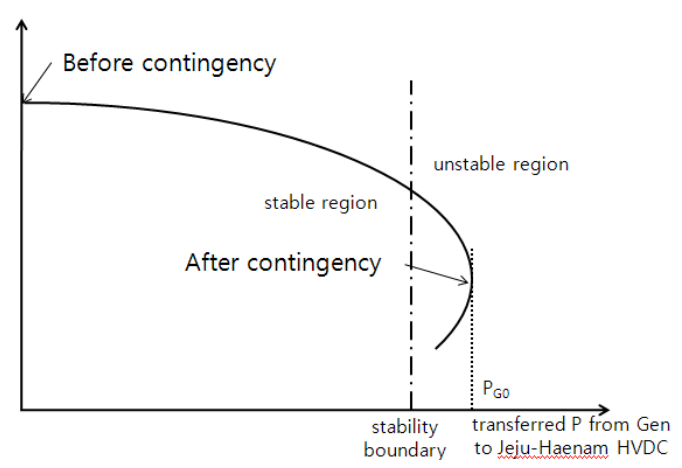

(b) unstable case

Fig. 3. Generator contingency analysis using CPF algorithm. 
is under fault condition. We can find that the solution path approaches the outage point by generation shift from faulted generators to HVDC system. As the output of the generator decreases, that is, transmission of active power in HVDC increases, consumption of reactive power in the system increases, whereby a nose curve shaped graph showing decrease of voltage is obtained. Figure 3(a) is a stable case where the capacity of faulted generator is smaller than the stability boundary, which can be determined by transferred power over the HVDC system. Figure 3 (b) is for the case that the capacity of faulted generator is larger than $\triangle \mathrm{PHVDC}$, which is an unstable case in which HVDC cannot cover PG0.

A method of solving the afore-mentioned problem is indicated in Fig. 4. Fig. 4(a) is limiting the output of the faulted generator for decreasing active power shifted from the generator to the HVDC. Fig. 4(b) is connecting reactive power source to system B for releasing the limitation of HVDC transmission capacity due to insufficient reactive power. Connection of a new source of reactive power requires certain procedure and time allowance. In this paper, methods of limiting the maximum output of the generator are handled. In Section II-B, a system modeling for realizing the algorithm to calculate the generation output limitation is handled.

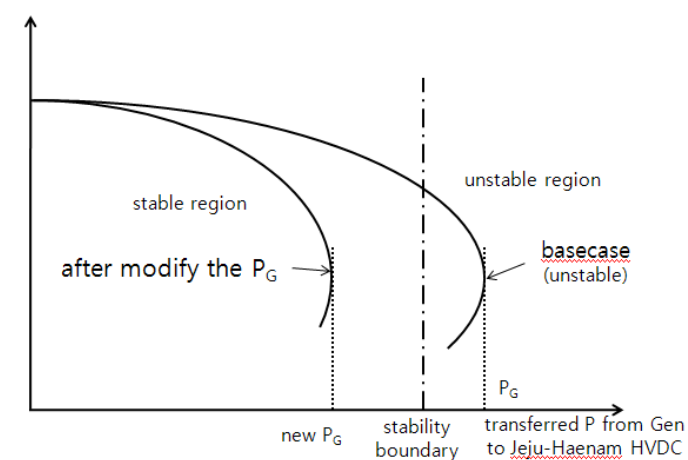

(a) Limitation of critical generator

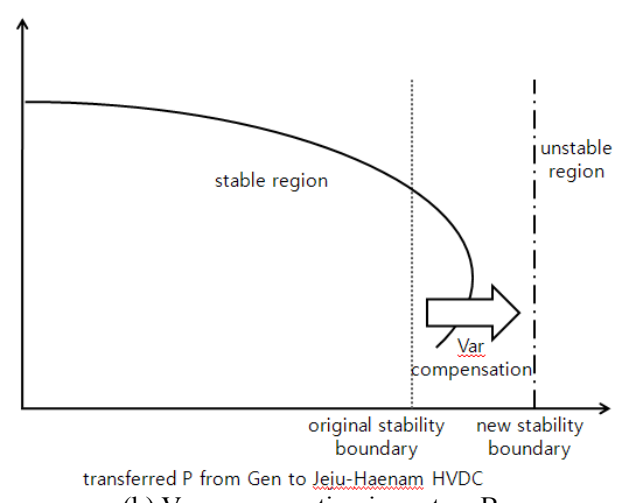

(b) Var compensation in system B

Fig. 4. Countermeasures to avoid voltage collapse.

\subsection{System Modeling}

In order to apply the CPF (Continuation Power Flow) method, a stress parameter (e.g., increasing loads) must be inserted into the power flow equations. In general, the power flow equations at bus $i$ are as follows:

$$
\begin{aligned}
& P_{T i}(\delta, V)-P_{G i}+P_{L i}=0 \\
& Q_{T i}(\delta, V)-Q_{G i}+Q_{L i}=0
\end{aligned}
$$

where the subscript $\mathrm{T}, \mathrm{G}, \mathrm{L}, \delta$, and $\mathrm{V}$ represent injection, generation, load, bus voltage angle, and bus voltage magnitude, respectively. In Fig. 2, a generator fault increases the transmission of HVDC power, and this eventually increases the demand for reactive power in the HVDC. To materialize this scenario, we express the generation shifted from the faulted generator to HVDC as stress parameter $\mu$.

We put the generation before fault as $\mathrm{P} 0$ and we know the value of $\mu$ will be 0 before fault and 1 after fault through normalization. The steady-state behavior of the power system will be observed by varying the parameter $\mu$ from 0 to 1 . This means that parameter $\mu$ is 0 for precontingency' and 1 for 'post-contingency'. After remodeling the faulted generator and HVDC for each $\mu$, the above equation (1) and equation (2) will be recomposed.

Generators are sources of not only active power but also reactive power supplied to the system. Therefore, PGi and QGi of the faulted generator due to the variation of parameter $\mu$ (Shifted power from faulted generator to HVDC) have to be included in the power flow equation. The $\mathrm{Q}$ value of a generator in the power flow equation calculated after power flow solution is not appropriate to be used as a continuation parameter. Accordingly, the value of $\mathrm{Q}$ limit, which is the capacity of supplying (or consuming) the reactive power, has been used instead of $Q$ value of a generator. The equations of a faulted generator related with variation of continuation parameter $(\mu)$ are as follows.

$$
\begin{aligned}
& P_{G i}=(1-\mu) \times P_{G 0 i} \\
& Q_{G i \_M A X}{ }^{*}=(1-\mu) \times Q_{G i \_M A X} \\
& Q_{G i_{-} M I N}{ }^{*}=(1-\mu) \times Q_{G i_{-} M I N}
\end{aligned}
$$

Where, $\mathrm{P}_{\mathrm{G} 0 \mathrm{i}}$ Output $\mathrm{P}$ of pre-fault generator

$\mathrm{Q}_{\mathrm{Gi} \_\mathrm{MAX}}{ }^{*}$ Maximum $\mathrm{Q}$ limit in the faulted generator

$\mathrm{Q}_{\mathrm{Gi} \text { MIN }}{ }^{*}$ Minimum $\mathrm{Q}$ limit in the faulted generator

$\mathrm{Q}_{\mathrm{Gi} \text { MAX }}$ Maximum Q limit in the pre-faulted generator

$\mathrm{Q}_{\mathrm{Gi} \_\mathrm{MIN}}$ Minimum Q limit in the pre-faulted generator

As the HVDC is operated on frequency control mode, it is evident that the power shifted from generators to HVDC system increases by $\mu$ PG0 when $\mu$ varies. Also, the HVDC will consume more reactive power of system $B$ in order to transmit additional power corresponding to $\mu \mathrm{PG}$. Generally, $50 \sim 60 \%$ of active power is consumed for the transmission of active power in steady-state condition. Under transient condition, more reactive power is required momentarily. Fig. 5 shows the measurement data of actual fault in the Jeju-Haenam HVDC system. Fig. 5(a) presents 
the measured result for variation of active power transmission $(\mathrm{P})$ and reactive power $(\mathrm{Q})$ consumed in HVDC by changing transmission power in the event of system frequency fluctuation due to the disturbance in the Jeju system.

From Fig. 5(b), we can find that approximately $68 \%$ of transmission power is consumed for reactive power in the steady-state condition and $80 \%$ of transmission power is consumed in the transient condition. It is assumed that generators supply during the transient period $(2 \sim 3 \mathrm{sec})$, as they are capable of supplying more power than the rated IFD(excitation current) for a short duration of time under the transient condition. In this paper, the HVDC system is represented as a minus load which supplies active power to the system. The HVDC system can be modeled as follows.

$$
\begin{gathered}
P_{H}=-\left(P_{H 0}+\mu P_{G 0}\right) \\
Q_{H}=\alpha \times P_{H 0}
\end{gathered}
$$

Where,

$\mathrm{P}_{\mathrm{H}}$ Active power transferred from HVDC by variation of $\mu$

$\mathrm{Q}_{\mathrm{H}} \quad$ Consumed Reactive power in HVDC system by variation of $\mu$

$\mathrm{P}_{\mathrm{H} 0}$ Active power transferred by HVDC before fault

$\alpha \quad \mathrm{Q} / \mathrm{P}$ ratio of the HVDC system

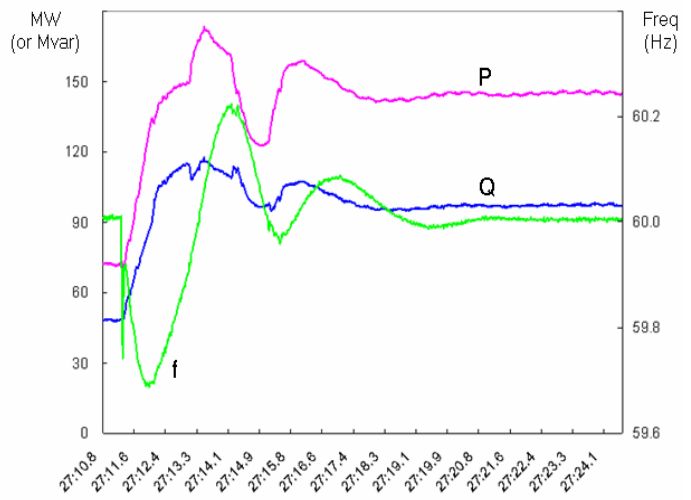

(a) Response of HVDC after fault

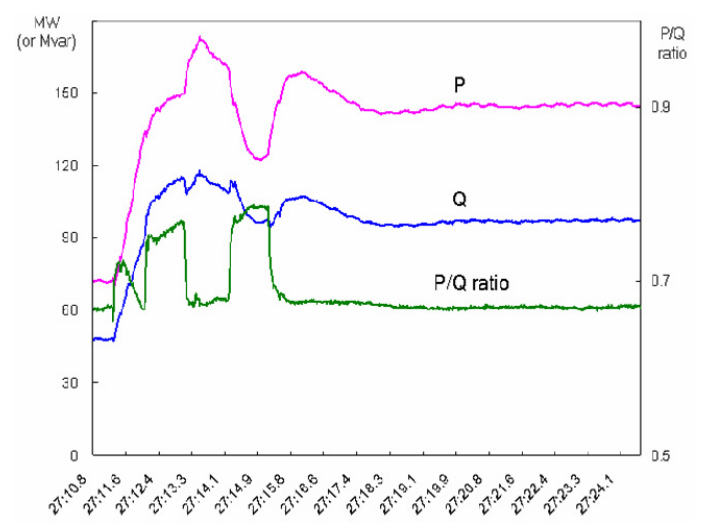

(b) Q consumption for power transfer

Fig. 5. The relationships between transfer active power and reactive power consumption in the Jeju-Haenam HVDC system.

\subsection{Methodology for Determining of Generator Opera- tion Point for Maintaining Stability}

This subsection elaborates on a method of deciding the new operation point of a generator for maintaining system stability when a new equilibrium point does not exist after a generator fault. (Also see Fig. 4.) In Fig. 6, information on the amount of maximum shift within the stability limits the attainment of power flow solution. A new operation point of a generator for maintaining stability can be acquired from equation (8).

$$
P_{G_{-} \lim }=P_{G 0} \times \mu_{M A X}
$$

Where, $\mathrm{P}_{\mathrm{G} 0}$ Original output of generator before limiting

$\mu$ MAX Maximum value of continuation parameter $\mu$

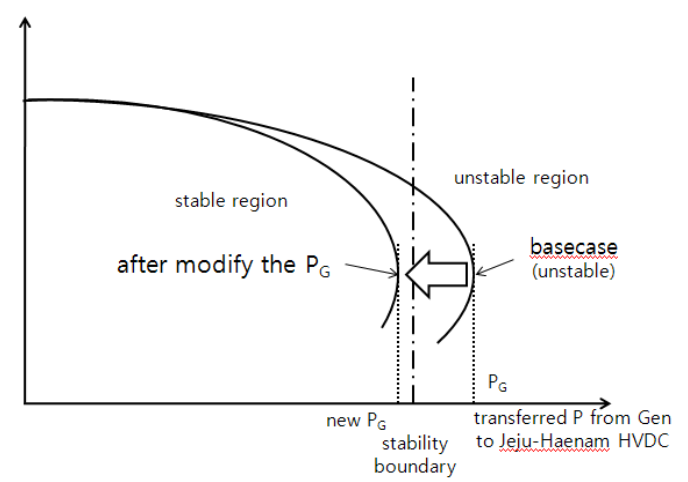

Fig. 6. Determining new PGout for system stability.

PG_lim becomes a new operation point of a generator for system stability, and the extra transmission capacity of the HVDC system is limited by PG_lim.

The procedure of deciding the generator operation limit (a maximum transmission power of HVDC) is as follows.

Step 1) Analyze the $P, Q$ ratio of HVDC and select the object group of generators.

Step 2) Perform modeling of faulted generator and HVDC, and execute the proposed method.

Step 3) Select unstable contingency in "Step 2". If the system is stable, the network system is considered stable.

Step 4) If the system is unstable, a new operation point of the generator is calculated using equation (8).

Step 5) Verify the stability through system analysis after limiting the generation capacity.

If the generation reserve for generation dispatch is not sufficient when limiting generation capacity in "Step 5", a new generator has to be operated considering generation cost.

\section{Result of the Application in Jeju System}

In this section, the simulation result is handled using the proposed method on the Jeju system. Table 2 shows the major generation data of the Jeju system. The total load of 
Jeju is approximately 500MW, and $150 \mathrm{MW}$ is supplied from HVDC. Operation of the HALLIM combined cycle power plant has been stopped as the operation cost is higher than those of the other plants. In Fig. 5(b), PQ ratio $(\alpha)$ is considered as 0.7 approximately.

Table 1. The status of generators in Jeju system

\begin{tabular}{c|c|c|c}
\hline \multirow{2}{*}{ Gen Name } & Case 1 & Case 2 & \multirow{2}{*}{$\begin{array}{c}\text { Pmax } \\
(\mathrm{MW})\end{array}$} \\
\cline { 2 - 3 } & $\mathrm{P}_{\mathrm{GEN}}(\mathrm{MW})$ & $\mathrm{P}_{\mathrm{GEN}}(\mathrm{MW})$ & 100 \\
\hline SJEJU TP\#3 & 100 & 95 & 100 \\
\hline SJEJU TP\#4 & 0 & 95 & 75 \\
\hline JEJU TP\#2 & 0 & 42 & 75 \\
\hline JEJU TP\#3 & 0 & 42 & 40 \\
\hline JEJU DP & 40 & 38 & \\
\hline
\end{tabular}

\subsection{Case 1(unstable case)}

During off-peak hours, $50 \%(140 \mathrm{MW})$ of the total load (280MW) is supplied from HVDC (see Table 1), and the remaining loads are supplied from SJEJU TP\#3 and JEJU $\mathrm{D} / \mathrm{P}$ considering the generation cost (economical efficiency). As only 2 generators are operated, the object generator groups for analysis are SJEJU TP\#3 and JEJU D/P. The result of CPF analysis after modeling on the respective $\mu$ for 2 generators and HVDC is shown in Fig. 7. Fig. 7(a) gives the result of a fault on SJEJU TP\#3. The system collapses before $\mu$ reaches 1 , which is an unstable case. Fig. 7(b) is the result of JEJU D/P fault.

The system solution exists once the $\mu$ becomes 1 , and this is judged to be a stable condition. The worst contingency is a fault on SJEJU TP\#3, and the generation limit is calculated using equation (8). The $\mu$ value on collapse of

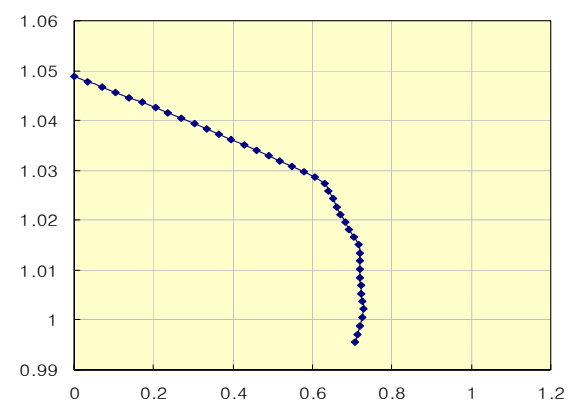

(a) Upon fault on SJEJU TP\#3

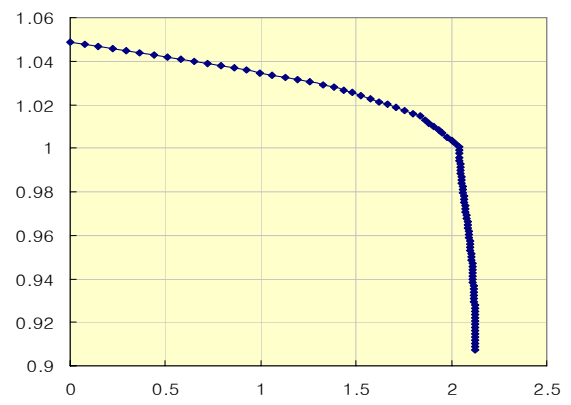

(b) Upon fault on SJEJU TP\#3

Fig. 7. Result of Generator Fault Analysis during Off-peak Load Demand. solution is 0.73 , and the new operation point of the generator is $73 \mathrm{MW}(0.73 \times 100 \mathrm{MW}=73 \mathrm{MW}) .27 \mathrm{MW}$ has to be supplied from another generator. As it is not possible to receive any more power from the two generators being operated in the Jeju system, operation of another generator has to be started. Fig. 8 is the result of CPF analysis after startup of JEJU TP\#2 considering economical efficiency. In Fig. 8 , the $\mu$ value reaches 1.2 exceeding 1.0 because the source of reactive power has increased by the paralleling of a new generator into the system. Actually, JEJU TP\#2 is capable of supplying 35Mvar of reactive power to the system.

As a new generator has to start operation when generation capacity is limited, the proposed algorithm has been executed again with a new generator (JEJU TP\#2) being operated. The result is indicated in Fig. 9, where the $\mu$ value is 0.95 , and the new output $(\mathrm{P})$ is $95 \mathrm{MW}(0.95 \mathrm{X}$ 100MW). Fig. 10 indicates the result of the proposed analysis

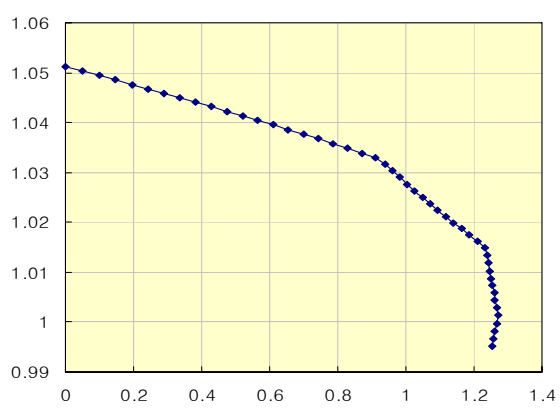

Fig. 8. Result of the proposed analysis after changing the generator operating point from $100 \mathrm{MW}$ to $73 \mathrm{MW}$.

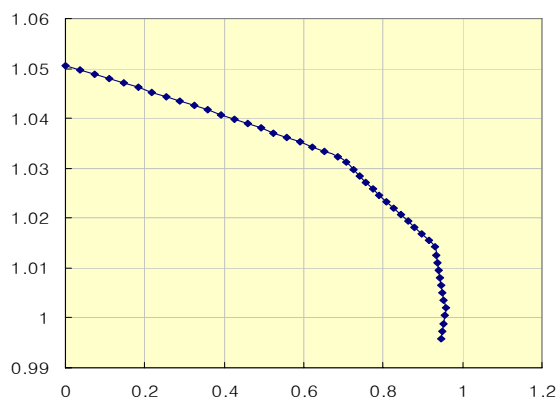

Fig. 9. Result of the propose analysis before limiting SJEJU TP\#3.

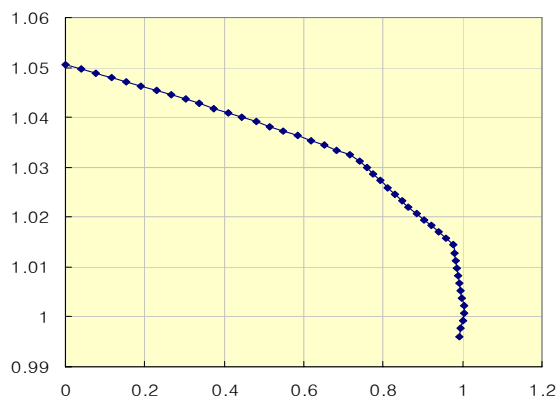

Fig. 10. Result of the proposed analysis after changing the generator operating point from $100 \mathrm{MW}$ to $95 \mathrm{MW}$. 
of a fault on SJEJU TP\#3 when SJEJU TP\#3 is limited to $95 \mathrm{MW}$ and JEJU TP\#2 generates the remaining $5 \mathrm{MW}$.

\subsection{Case 2(stable case)}

During peak hours in the Jeju system, total load is approximately 530MW, of which HVDC supplies $150 \mathrm{MW}$ and the remaining loads are supplied by the other generators in the system (Table 2). Both $\mathrm{P}$ ratio and $\mathrm{Q}$ ratio of the HVDC system are considered as 0.7 . The assumed faulted generators are SJEJU TP\#3, JEJU TP\#2, and JEJU D/P. The result of the proposed analysis on the most sever contingency (SJEJU TP\#3) is indicated in Fig. 11. For the moment when $\mu$ is 1.0 , i.e. the moment of fault, power flow solution exists for each assumed fault. As there are many generators under operation in the Jeju system during peak demand, reactive power reserve is sufficient in the system different from off-peak demand. This explains the reason why the system is stable when HVDC transmits all the power corresponding to the output of a generator disconnected from the system.

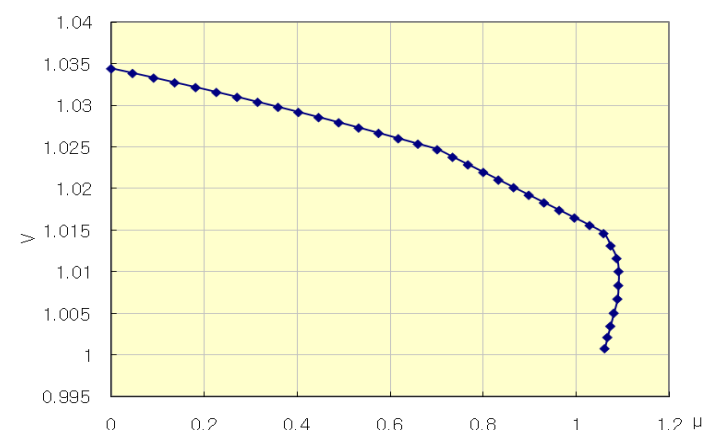

Fig. 11. Result of the proposed analysis upon fault in SJEJU TP\#3 (the most sever contingency).

\section{Conclusion}

As the Jeju-Haenam HVDC system is operated on frequency control mode, the HVDC system maintains the system frequency when out-of-step occurs with large generators in the Jeju system. And, it was recognized that the Jeju system could be unstable if the reactive power consumed by the HVDC is insufficient for the Jeju system. When there is no solution of power flow due to the unstable system phenomenon, we have difficulty in establishing countermeasures. For the purpose of overcoming this difficulty, introduction of the CPF algorithm is intended in this paper. In order to apply the CPF method, we express the generation shifted from the faulted generator to HVDC as stress parameter $\mu$ and recompose the power flow equations. The HVDC system is represented as a minus load which supplies power to the system. We suggest the new operation point of a generator for system stability when a new equilibrium point does not exist after a generator fault in this paper.

\section{Acknowledgements}

This work was the outcome of a Manpower Development Program for Energy for \& Resources supported by the Ministry of Knowledge and Economy (MKE)

\section{References}

[1] B.-E. Koh, G.-J. Jung, I.-H. Moon, S.-K. Kim, "Introduction of Haenam-Jeju HVDC system," IEEE International Symposium on Industrial Electronics, pp.1006-1010, 2001.

[2] G. Jang, S. Oh, B.M. Han, C.K. Kim, "Novel reactivepower-compensation scheme for the Jeju-Haenam HVDC system," IEE Proceedings: Generation, Transmission and Distribution, pp.514-520, July, 2005.

[3] Byeong-Mo Yang, Chan-Ki Kim, Gil-Jo Jung, YoungHyun Moon, "Verification of Hybrid Real Time HVDC Simulator in Cheju-Haenam HVDC Systems," Journal of Electrical Engineering \& Technology, Vol.1, No.1, Mar., 2006.

[4] T Smde, G Andersson, "Analysis of power/voltage stability of HVDC converters in AC systems," AC and DC Power Transmission, International Conference on 17-20, pp.395-397, Sep., 1991

[5] K R Padiyar, S S Rao, "Dynamic analysis of voltage instability in AC-DC systems," Electrical Power \& Energy Systems Vol.18, No.1, pp.11-18, 1996.

[6] R. S. Thallam, "Review of the design and performance features of HVDC systems connected to low short circuit ratio AC systems," IEEE Trans, Vol7, pp.2065-2073, 1992

[7] Chiang H-D., Flueck A.J, Shah K.S, Balu N, "CPFLOW: A Practical Tool for Tracing Power System Steady-State Stationary Behavior Due to Load and Generation Variations," IEEE Trans, Vol.10, No.2, May 1995.

[8] Venkataramana Ajjarapu, Colin Christy. "The Continuation Power Flow: A Tool for Steaty State Voltage Stability Analysis," IEEE Trans, 1991.

[9] Alexander J. Flueck, Jayabharath R. Dondeti, “A New Continuation Power Flow Tool for Investigating the Nonlinear Effects of Transmission branch Parameter Variations," IEEE Trans, Vol.15. No.1, FEB., 2000.

[10] H. Song, S.-D. Baik, B. Lee, "Determination of load shedding for power-flow solvability using outagecontinuation power flow(OCPF)," IEEE Proceedings: Generation, Transmission and Distribution, Vol.1543, No.3, May 2006.

[11] Lee B., Song H., Kwon S.-H., Jang G., Kim J.-H., Ajjarapu V, "A Study on Determination of Interface Flow Limits in the KEPCO system Using Modified Continuation Power Flow (MCPF)", IEEE Trans, Vol.17, No. 3, August, 2002

[12] Lee B., Song H., "A Special Protection Scheme against a Local Low-Voltage Problem and Zone 3 
Protection in the KEPCO System", Journal of Electrical Engineering \& Technology, Vol.2, No.3, Sep., 2007

[13] Prabha Kundur, "Power System Stability and Control", McGraw-Hill, Inc.

[14] Thierry Van Cutsem, "Voltage Stability of Electric Power System", Kluwer Academic Publishers.

[15] Carson W. Taylor, "Power system voltage stability", McGraw-Hill, Inc.

[16] Venkataramana Ajjarapu, "Computational Techniques for Voltage Stability Assessment and Control," Springer.

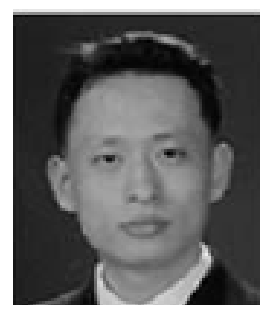

Sang-Gyun Kang received the B.S. and M.S. degrees in Electrical Engineering, from Korea University, Seoul, Korea, in 2003 and 2005, respectively, where he currently pursuing the Ph.D. degree. His research interests include voltage stability and control in power systems.

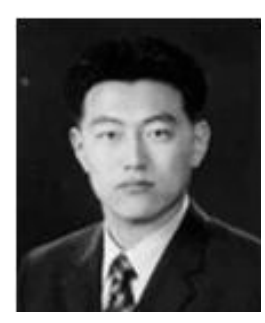

Sangsoo Seo received B.S. and M.S. degrees in Electrical Engineering from Korea University, Seoul, Korea in 2003 and 2005 respectively. He is currently a Ph.D.'s course student in the Dept. of Electrical Engineering at Korea University. His interests include power system operation, voltage stability indices and hybrid voltage control.

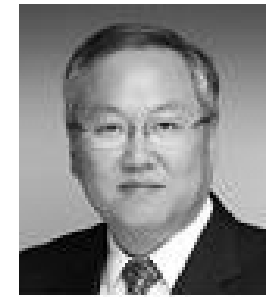

Byongjun Lee received B.S. degrees in Electrical Engineering from Korea University, Seoul, Korea in 1987, M.S. and Ph.D. degrees in Electrical Engineering from Iowa State University in 1991 and 1994 respectively. He is currently a professor in the Dept. of Electrical Engineering at Korea University. His interests include power system operation, voltage control, system protection schemes (SPS), FACTS equipment and PMU.

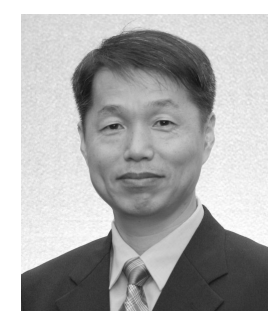

Joon-Young Joo received B.S. degrees in Electrical Engineering from Chonbuk University, Jeonju, Korea in 1986, He is currently a deputy manager in the Dept. of System Operation and Control at the KPX (Korea Power eXchange). His interests include power system operation, and demand /supply planning. 